\title{
A SUFFICIENT CONDITION FOR TOTAL MONOTONICITY
}

BY

\author{
B. E. RHOADES
}

Let $\mu=\left\{\mu_{n}\right\}$ be a sequence of real numbers. Then $\mu$ is said to be totally monotone if and only if all of the successive forward differences are nonnegative; i.e., $\Delta^{n} \mu_{k} \geqq 0$ for $k, n=0,1,2, \cdots$, where $\Delta \mu_{k}=\mu_{k}-\mu_{k+1}, \Delta^{n} \mu_{k}=\Delta^{n-1} \mu_{k}-\Delta^{n-1} \mu_{k+1}$.

There are several equivalent necessary and sufficient conditions for a sequence to be totally monotone. These are listed in $\$ 1$. However, these conditions are often quite difficult to apply to a given sequence or class of sequences.

It is the purpose of this paper to establish a sufficient condition for a sequence to be totally monotone, and show that, for a fairly large class of sequences, the condition is more easily applied than those listed in $\$ 1$.

1. Some conditions for total monotonicity. The first phrasing is merely the continuous analogue to that for sequences, and is referred to in [11, p. 145] as a completely monotonic function. However, a function satisfying the condition (F) stated below will be called a totally monotone function.

Let $f(t)$ be an analytic function of $t>0$ such that $f(n)=\mu_{n}$. Then the sequence $\mu$ is totally monotone if and only if

$$
(-1)^{n} f^{(n)}(t) \geqq 0
$$

for $t>0, n=0,1,2, \cdots$, and $f(0) \geqq f(0+)$.

Hausdorff [6] established the following condition.

Let $\mu$ denote a sequence of real numbers. Then $\mu$ is totally monotone if and only if

$$
\mu_{n}=\int_{0}^{1} u^{n} d \phi
$$

where $\phi(u)$ is a nonnegative, nondecreasing function of bounded variation over the interval $[0,1]$ with $\phi(0+)$ $=\phi(0)$, and, for $0<u<1, \phi(u)=[\phi(u+0)+\phi(u-0)] / 2$.

The function $\phi$ is usually normalized so that $\phi(1)=1$, and $\phi$ is called the mass function corresponding to the sequence $\mu$.

Presented to the Society, January 25, 1961 and January 23, 1962, under the respective titles Some sets of totally monotone sequences and $A$ sufficient condition for total monotonicity; received by the editors May 9, 1962. 
H. S. Wall [10] in 1940 proved the following continued fraction condition for total monotonicity.

A sequence $\mu$ is totally monotone if and only if there exist real numbers $g_{0}$, $g_{1}, g_{2}, \cdots$ with $0 \leqq g_{n} \leqq 1, n=0,1,2, \cdots$, such that

$$
\begin{aligned}
\mu_{0}-\mu_{1} x+\mu_{2} x^{2}-\mu_{3} x^{3}+\cdots \sim g_{0} / 1 & +g_{1} x / 1+\left(1-g_{1}\right) g_{2} x / 1 \\
& +\left(1-g_{2}\right) g_{3} x / 1+\cdots
\end{aligned}
$$

it being agreed that the continued fraction shall terminate with the first identically vanishing quotient.

In 1921 Hausdorff [5] established the following sufficient condition for total monotonicity.

Let $f(t)$ be an analytic function for $t>0$. Write $f(t)$ as $e^{\sigma(t)}$, where $\sigma(t)=\log f(t)$. If $(-1)^{n} \sigma^{(n)}(t) \geqq 0$ for $t \geqq 0$ and $\sigma(0) \geqq \sigma(0+)$, then $(-1)^{n} f^{(n)}(t) \geqq 0$.

Rephrasing the above condition leads to the following result, which is listed, since it will be referred to explicitly later.

LEMMA 1. Let $\mu$ be a nonnegative sequence such ithat $\Delta \mu_{n}=\mu_{n} \alpha_{n}$, where $\alpha=\left\{\alpha_{n}\right\}$ is a totally monotone sequence, $n=0,1,2, \cdots$. Then $\mu$ is totally monotone.

Equivalently, let $\mu(t) \geqq 0$ be an analytic function for $t>0$ and such that $(-1) \mu^{\prime}(t)=\mu(t) f(t)$. If $f(t)$ is a totally monotone function, then $\mu(t)$ is a totally monotone function.

2. Some sets of totally monotone sequences. It is the purpose of this section to lay the groundwork for the sufficient condition for total monotonicity that is to be utilized in the next section.

Let $T$ denote the set of totally monotone sequences, $L=\{\mu \mid \mu \in T$ and $\Delta^{n} \log \mu_{k} \geqq 0$ for $\left.n=1,2,3, \cdots ; k=0,1,2, \cdots\right\}$, and $Q=\{\mu \mid \mu \in T$ and $\left.\left\{\mu_{k} / \mu_{k+1}\right\} \in T\right\}$.

In defining $L$ and $Q$ it is the purpose to have them be subsets of $T$. It is clear that $L$ is the set of sequences which correspond to the functions satisfying condition (L) of $\S 1$. The following properties of $L$ and $Q$ are easily established.

(i) $L \cap Q$ is nonempty.

(ii) $L$ and $Q$ are commutative semigroups with respect to sequence multiplication.

Property (ii) is obvious, and (i) is easy to show, since the intersection contains the Cesàro and Hölder generating sequences of all positive orders.

The set $T$ consists of two kinds of sequences: those of the form $\{c, 0,0, \cdots\}, c \geqq 0$ and those with no zero entries. Let $N=\{\mu \mid \mu=\{c, 0,0, \cdots\}, c \geqq 0\}$. Define a 
family of quotient operations on $T \sim N=\{\mu \mid \mu \in T$ and $\mu \notin N\}$ as follows : $q_{1}\left(\mu_{k}\right)=\mu_{k} / \mu_{k+1}, \cdots, q_{n}\left(\mu_{k}\right)=q_{1}\left(q_{n-1}\left(\mu_{k}\right)\right)$. Let $Q_{n}$ denote the set of totally monotone sequences whose first $n$ "quotients" are not less than 1 ; i.e., $\mu \in Q_{n}$ implies $q_{r}\left(\mu_{k}\right) \geqq 1, k=0,1,2 \cdots ; r=1, \cdots, n$. From the definition of the $Q_{n}$, $T \supset Q_{1} \supset Q_{2} \supset \cdots$. Let $Q_{\infty}=\bigcap_{i=1}^{\infty} Q_{i}$.

(iii) $Q_{1}$ is a proper subset of $T$.

(iv) The operator $q_{n}$ is commutative; i.e., $q_{n}\left(q_{m}\left(\mu_{k}\right)\right)=q_{m}\left(q_{n}\left(\mu_{k}\right)\right), m, n$ $=0,1,2, \cdots$.

(v) $Q_{1}=Q_{2}$

(vi) $Q$ is a proper subset of $Q_{3}$.

(vii) $L=Q_{\infty}$.

(viii) $\mu \in Q_{\infty}$ if and only if $q_{n}\left(\mu_{k}\right)$ is totally monotone for each $n$.

(ix) $L \subset Q$.

(x) Let $\mu$ be a sequence such that $\left\{\mu_{k} / \mu_{k+1}\right\}$ is totally monotone. Then $\mu \in T$ if and only if $\mu_{k} \geqq \mu_{k+1}$.

(xi) There exists an integer $m>2$ such that $Q_{m+1}$ is a proper subset of $Q_{m}$.

Properties (iii) and (iv) are obvious. To prove (v), first note that $Q_{1}=T \sim N$. Then, from [10, (5.11)], we have $\left(\Delta \mu_{k} / \mu_{k}\right)<\left(\Delta^{2} \mu_{k} \Delta \mu_{k}\right)$ which can be rewritten in the form $\mu_{k} \mu_{k+2}-\left(\mu_{k+1}\right)^{2}>0$; i.e., $q_{2}\left(\mu_{k}\right)>1$. Then property (v) follows, since $Q_{2} \subset Q_{1}$.

For (vi), consider the sequence $\mu_{k}=1+e^{-k}$. It is easy to show that $q_{3}\left(\mu_{k}\right) \geqq 1$. However, if we let $f(t)=\left(1+e^{-t}\right) /\left(1+e^{-t-1}\right)$, then $(-1) f^{\prime \prime \prime}(t)<0$ for $0<t<-1+\log (2+\sqrt{ } 3)$, and thus $\mu \notin Q$.

To prove (vii), note that $\Delta \log \mu_{k}=\log \left(\mu_{k} / \mu_{k+1}\right)=\log q_{1}\left(s_{k}\right)$ and, by induction, that $\Delta^{n+1} \log \mu_{k}=\log q_{n}\left(\mu_{k}\right)$. Therefore $\Delta^{n+1} \log \mu_{k} \geqq 0$ if and only if $q_{n}\left(\mu_{k}\right) \geqq 1$.

Proof of (viii). Let $\mu \in Q_{\infty}$. Then $\Delta q_{n}\left(\mu_{k}\right)=q_{n}\left(\mu_{k+1}\right)\left[q_{n+1}\left(\mu_{k}\right)-1\right] \geqq 0$, and, in general,

$$
\Delta^{r} q_{n}\left(\mu_{k}\right)=\sum_{j=0}^{r-1}\left(\begin{array}{c}
r-1 \\
j
\end{array}\right)\left[\Delta^{r-1-j} q_{n}\left(\mu_{k+j+1}\right)\right]\left[\Delta j\left\{q_{n+1}\left(\mu_{k}\right)-1\right\}\right] \geqq 0 .
$$

Conversely, $q_{n}\left(\mu_{k}\right)$ totally monotone for each $n$ implies $\Delta q_{n}\left(\mu_{k}\right) \geqq 0$. But $\Delta q_{n}\left(\mu_{k}\right)=q_{n}\left(\mu_{k+1}\right)\left[q_{n+1}\left(\mu_{k}\right)-1\right]$. Therefore $q_{n+1}\left(\mu_{k}\right) \geqq 1$; i.e., $\mu \in Q_{n+1}$ for each $n$.

Property (ix) follows directly from (vii) and (viii), (x) is straightforward, and, for (xi), if we assume the contrary, we arrive at a contradiction of (ix).

For the purpose of this paper, properties (ix) and (x) are the most fruitful. Property ( $\mathrm{x}$ ) gives a sufficient condition for a sequence to be totally monotone, and (ix) says that this condition is sufficient for a class of sequences at least as large as those satisfying condition (L).

In applying this condition we shall use the procedure outlined in the following theorem. 
THEOREM 1. Let $\mu=\left\{\mu_{n}\right\}$ be a real positive sequence, $f(t)$ a function of class $C^{\infty}$ for $t>0$ such that $f(k)=\mu_{k}, k=0,1,2, \cdots, g(t)=f(t) / f(t+1)$, $h(t)=(-1) g^{\prime}(t) / g(t)$. If $\lim _{t \rightarrow \infty} g(t) \geqq 1, g(t)>0$ and $h(t)$ is totally monotone for $t>0$, then $\mu$ is a totally monotone sequence.

To prove the theorem, since $g(t)>0$ and $h(t)$ is totally monotone, then $g(t)$ will be totally monotone by Lemma 1 . Hence $f(t) \downarrow$ in $t$. Since $\lim _{t \rightarrow \infty} g(t) \geqq 1$, then $f(t) / f(t+1) \geqq 1$. Therefore, by property $(\mathrm{x}), \mu$ is totally monotone.

Before proceeding to the applications we prove a lemma, which will be used repeatedly.

Lemma 2. Let $\mu_{n}=(n+a+\alpha)(n+b) /(n+a)(n+b+\alpha), a, b>0$. Then $\mu$ is totally monotone if and only if

$$
\alpha \geqq 0 \text { and } b \geqq a
$$

$$
\alpha<0, b<a \text { and } b+\alpha>0 \text {. }
$$

Proof of sufficiency. Let $f(t)=(t+a+\alpha)(t+b) /(t+a)(t+b+\alpha)$. Then, if

$$
g(t)=(-1) \frac{f^{\prime}(t)}{f(t)}=\frac{1}{t+a}+\frac{1}{t+b+\alpha}-\frac{1}{t+a+\alpha}-\frac{1}{t+b},
$$

the mass function corresponding to $g(t)$ is

$$
\phi(u)=\frac{u^{a}}{a}+\frac{u^{b+\alpha}}{b+\alpha}-\frac{u^{a+\alpha}}{a+\alpha}-\frac{u^{b}}{b}, \quad 0 \leqq u \leqq 1 .
$$

For $0<u<1$,

$$
\begin{aligned}
\frac{d \phi}{d u} & =u^{a-1}+u^{b+\alpha-1}-u^{a+\alpha-1}-u^{b-1} \\
& =u^{a-1}\left(1-u^{\alpha}\right)\left(1-u^{b-a}\right) .
\end{aligned}
$$

Examination of conditions (A) and (B) shows that either condition implies $d \phi / d u \geqq 0$ for $0<u<1$. Therefore, by condition (M) of $\S 1, g(t)$ is totally monotone, and $f(t)$ is totally monotone by Lemma 1 .

Proof of necessity. Assume that (A) and (B) are not satisfied. Then we have the following possibilities to consider:
(a) $b<a$ and $\alpha \geqq 0$,
(b) $\alpha<0$ and $b \geqq a$,
(c) $\alpha<0$ and $b+\alpha \leqq 0$, or
(d) $b<a$ and $b+\alpha \leqq 0$.

It is clear that $f(t)$ cannot be totally monotone if there exists a value of $t>0$ such that $g(t)<0$. We may rewrite $g(t)$ in the form 


$$
g(t)=\frac{\alpha(b-a)(2 t+b+a+\alpha)}{(t+a)(t+b+\alpha)(t+a+\alpha)(t+b)} .
$$

By inspection, each of the conditions (a) through (d) makes $g(t)$ negative for some positive $t$.

3. Applications. Let $x$ denote a sequence, $A$ an infinite matrix for which $A_{n}(x)=\sum_{k} a_{n k} x_{k}$ is defined. $A$ is said to be regular if $x_{n} \rightarrow l$ implies $A_{n}(x) \rightarrow l$, $l$ finite. For two regular matrices $A$ and $B, B$ is said to be totally stronger than $A$ (written $B$ t.s. $A$ ) if, for each sequence $x$ for which $A_{n}(x) \rightarrow l$, then $B_{n}(x) \rightarrow l$ $(|l| \leqq \infty)$. If $A$ and $B$ are regular matrices for which $A_{n}(x) \rightarrow l$ implies $B_{n}(x) \rightarrow l, l$ finite, but there exists a sequence $x \operatorname{such} A_{n}(x) \rightarrow+\infty$ but $B_{n}(x) \rightarrow+\infty$, then we say that $B$ is not totally stronger than $A$ (written $B$ n.t.s. $A$ ). The definition of not totally stronger is also meant to include the case where $A_{n}(x) \rightarrow-\infty$ and $B_{n}(x) \rightarrow-\infty$. In all cases where one is determining the total relative strength of two matrices $A$ and $B$, one must first have the condition that $B$ is stronger than $A$; i.e., $A_{n}(x) \rightarrow l$ implies $B_{n}(x) \rightarrow l$ for $l$ finite.

If $H_{\mu}$ and $H_{\lambda}$ denote the Hausdorff matrices generated by the sequences $\mu$ and $\lambda$, and if $\lambda_{n} \neq 0$ for any $n$, then the statement $H_{\mu}$ t.s. $H_{\lambda}$ reduces to showing that the sequence $\left\{\mu_{n} / \lambda_{n}\right\}$ is totally monotone and that $\mu_{0}=\lambda_{0}$.

Let $H_{\mu}$ and $H_{\lambda}$ be two Hausdorff matrices which are equivalent; that is, $H_{\mu}$ is stronger than $H_{\lambda}$ and $H_{\lambda}$ is stronger than $H_{\mu}$. Then, to show that $H_{\mu}$ n.t.s. $H_{\lambda}$ it is sufficient to show that $\left\{\mu_{n} / \lambda_{n}\right\}$ is not totally monotone.

For the other terminology used in this section, the reader should consult $[1 ; 3$; or 7], and for the basic properties of Hausdorff matrices, see [4, XI].

Let $H^{\alpha}, \Gamma_{a}^{\alpha}, C_{a}^{\alpha}$ denote the methods generated, respectively, by the sequences $(n+1)^{-\alpha}, a^{\alpha}(n+a)^{-\alpha}$ and $\Gamma(a+\alpha) \Gamma(n+a) /[\Gamma(a) \Gamma(n+a+\alpha)]$, for $a>0$, $\alpha>-1$. $H^{\alpha}$ is the Hölder method of order $\alpha, \Gamma_{a}^{\alpha}$ is the Gamma method of order $\alpha$, and $C_{a}^{\alpha}$ for $a=1$, reduces to the Cesàro method of order $\alpha$.

$H^{\alpha}$ and $C_{1}^{\alpha}$ have been compared totally in [1], and $H^{\alpha}, C_{1}^{\alpha}, \Gamma_{a}^{\alpha}$ have been compared totally in [7]. It is the purpose of this section to examine the total relative strength of the $C_{a}^{\alpha}$ method, and then compare it with the $H^{\alpha}$ and $C_{1}^{\alpha}$ methods.

THeOREM 2. (i) For $\beta>\alpha, a+\alpha>0, C_{a}^{\beta}$ t.s. $C_{a}^{\alpha}$.

(ii) For $\alpha<0, a+\alpha>0, a<b, C_{b}^{\alpha}$ t.s. $C_{a}^{\alpha}$.

(iii) For $\alpha>0,0<a<b, C_{a}^{\alpha}$ t.s. $C_{b}^{\alpha}$.

Proof of (i). Let

Then

$$
\mu_{n}=\frac{\Gamma(a+\beta) \Gamma(n+a)}{\Gamma(a) \Gamma(n+a+\beta)} \cdot \frac{\Gamma(a) \Gamma(n+a+\alpha)}{\Gamma(a+\alpha) \Gamma(n+a)} .
$$

$$
\frac{\mu_{n}}{\mu_{n+1}}=\frac{(n+a+\beta)}{(n+a+\alpha)},
$$

and $\mu$ is totally monotone since $\beta>\alpha, a+\alpha>0$. 
Parts (ii) and (iii) are proved using Lemma 2.

THEOREM 3. (i) For $-1<\alpha, \beta<0, a+\alpha+\beta>0, C_{a}^{\alpha} C_{a}^{\beta}$ t.s. $C_{a}^{\alpha+\beta}$.

(ii) For $-1<\alpha, \beta, \alpha \beta<0, a+\min (\alpha, \beta)>0, C_{a}^{\alpha+\beta}$ t.s. $C_{a}^{\alpha} C_{a}^{\beta}$.

(iii) For $\alpha, \beta, a>0, C_{a}^{\alpha} C_{a}^{\beta}$ t.s. $C_{a}^{\alpha+\beta}$.

Proof of (ii). Let

$$
\mu_{n}=\frac{\Gamma(a+\alpha+\beta) \Gamma(n+a)}{\Gamma(a) \Gamma(n+a+\alpha+\beta)} \cdot \frac{\Gamma(a) \Gamma(n+a+\alpha)}{\Gamma(a+\alpha) \Gamma(n+a)} \cdot \frac{\Gamma(a) \Gamma(n+a+\beta)}{\Gamma(a+\beta) \Gamma(n+a)} .
$$

Then

$$
\frac{\mu_{n}}{\mu_{n+1}}=\frac{(n+a+\alpha+\beta)(n+a)}{(n+a+\alpha)(n+a+\beta)},
$$

and $\mu$ is totally monotone by Lemma 2 . (i) and (iii) are proved similarly. Theorem 3 generalizes [2, Theorem 1$]$.

THEOREM 4. (i) For $-1<\alpha<0, a+\alpha>0$, $\Gamma_{a}^{\alpha}$ t.s. $C_{a}^{\alpha}$.

(ii) For $0<\alpha<1, a>0, C_{a}^{\alpha}$ t.s. $\Gamma_{a}^{\alpha}$.

(iii) For $\alpha>1, a>0, \Gamma_{a}^{\alpha}$ t.s. $C_{a}^{\alpha}$.

Proof of (i) and (ii). Let

$$
\begin{aligned}
\mu_{n} & =\frac{a^{\alpha}}{(n+a)^{\alpha}} \frac{\Gamma(n+a+\alpha) \Gamma(a)}{\Gamma(a+\alpha) \Gamma(n+a)}, \\
\frac{\mu_{n}}{\mu_{n+1}} & =\left(\frac{n+a+1}{n+a}\right)^{\alpha}\left(\frac{n+a}{n+a+\alpha}\right) .
\end{aligned}
$$

Using the procedure outlined in Theorem 1, let

$$
g(t)=\frac{(t+a+1)^{\alpha}}{(t+a+\alpha)(t+a)^{\alpha-1}} .
$$

Then

$$
h(t)=\frac{-g^{\prime}(t)}{g(t)}=\frac{1}{t+a+\alpha}+\frac{\alpha-1}{t+a}-\frac{\alpha}{t+a+1},
$$

and $h(t)$ has as mass function

$$
\phi(u)=\frac{u^{a+\alpha}}{a+\alpha}+\frac{(\alpha-1) u^{a}}{a}-\frac{\alpha u^{a+1}}{a+1}, \quad 0 \leqq u \leqq 1 .
$$

For $0<u<1$,

$$
\frac{d \phi}{d u}=u^{a+\alpha-1}+(\alpha-1) u^{a-1}-\alpha u^{a}=u^{a-1} m(u),
$$

where $m(u)=u^{\alpha}+(\alpha-1)-\alpha u$. $d m / d u=\alpha\left(u^{\alpha-1}-1\right)$. If $-1<\alpha<0$ or 
$\alpha>1, d m / d u<0$. Therefore $m(u) \downarrow$ in $u$. But $m(1)=0$. Hence $\phi$ is totally monotone, and the results follow from Theorem 1 .

For $0<\alpha<1$ we consider $1 / \mu_{n}$. Using the same procedure as above we can show that the corresponding mass function is totally monotone and therefore $\left\{1 / \mu_{n}\right\}$ is totally monotone by Theorem 1 .

\section{THEOREM 5.}

(i) For $-1<\alpha<0, b>1, C_{b}^{\alpha}$ t.s. $H^{\alpha}$.

(ii) For $0<\alpha<1, b>1, C_{b}^{\alpha}$ t.s. $H^{\alpha}$.

(iii) For $\alpha>1,0<a<1, H^{\alpha}$ t.s. $C_{a}^{\alpha}$.

Let

Let

$$
\mu_{n}=\frac{\Gamma(b+\alpha) \Gamma(n+\alpha)}{\Gamma(b) \Gamma(n+b+\alpha)} \cdot \frac{(n+1)^{\alpha}}{1}
$$

$$
\begin{aligned}
g(t) & =\frac{(t+1)^{\alpha}(t+b+\alpha)}{(t+2)^{\alpha}(t+\alpha)} \\
h(t) & =(-1) \frac{g^{\prime}(t)}{g(t)}=\frac{\alpha}{t+2}+\frac{1}{t+\alpha}-\frac{\alpha}{t+1}-\frac{1}{t+b+\alpha}, \\
\phi(u) & =\frac{\alpha u^{2}}{2}+\frac{u^{\alpha}}{x}-\alpha u-\frac{u^{b+\alpha}}{b+\alpha}, 0 \leqq u \leqq 1 .
\end{aligned}
$$

For $0<u<1$,

$$
\begin{aligned}
\frac{d \phi}{d u} & =\alpha u+u^{\alpha-1}-\alpha-u^{h+\alpha-1} \\
& =u^{\alpha-1}\left[\alpha u^{1-\alpha}(u-1)+1-u^{b}\right] \\
& =u^{\alpha-1}\left[\left(1-u^{b}\right)-\alpha u^{1-\alpha}(1-u)\right]
\end{aligned}
$$

Proof of (i). Since $\alpha<0$, clearly $d \phi / d u>0$ and $h(t)$ is a totally monotone mass function.

Proof of (ii). Since $0<\alpha<1,1>\alpha u^{1-\alpha}>0$. Since $b>1$ and $0<u<1$, $1-u^{b}>1-u>0$. Therefore $1-u^{b}>\alpha u^{1-\alpha}(1-u)$ and thus $d \phi / d u>0$ for $0<u<1$.

Proof of (iii). From the construction of $h(t)$ it follows that the corresponding $h$-function for $1 / \mu_{n}$ is simply $-h(t)$.

Now consider the corresponding mass function $-\phi(u)$ for $\alpha>1$, with $b$ replaced by $a$.

$$
\frac{d(-\phi)}{d u}=\alpha(1-u)-u^{\alpha-1}\left(1-u^{a}\right)
$$

Since $\alpha>1,0<u<1, \alpha>u^{\alpha-1}>0$. Since $0<a<1,1-u>1-u^{a}>0$. Therefore $\alpha(1-u)>u^{\alpha-1}\left(1-a^{\alpha}\right)$ and $-\phi(u)$ is the mass function of a totally monotone function. 
Combining the results of Theorems 2,4 , and 5 with the statements R(i), (ii), and (iii) of [9, p. 397], we have the following list.

Let $0<a, a^{\prime}<1, b>1$. Then,

$$
\begin{aligned}
& \text { for } 0<a \leqq(\alpha+1) / 2<1+\alpha \leqq a^{\prime}<1, \\
& \Gamma_{b}^{\alpha} \text { t.s. } C_{b}^{\alpha} \text { t.s. } H^{\alpha} \text { t.s. } \Gamma_{a}^{\alpha} \text { t.s. } C_{a}^{\alpha}, \text { t.s. } C_{1}^{\alpha} \text { t.s. } \Gamma_{a}^{\alpha} \text { t.s. } C_{a}^{\alpha} \text {; } \\
& \text { for } 0<a \leqq(\alpha+1) / 2<1, \\
& C_{a}^{\alpha} \text { t.s. } \Gamma_{a}^{\alpha} \text { t.s. } C_{1}^{\alpha} \text { t.s. } C_{b}^{\alpha} \text { t.s. } H^{\alpha} \text { t.s. } \Gamma_{b}^{\alpha} ; \\
& \text { and, for } \alpha>1,2 b \geqq \alpha+1, \\
& \Gamma_{a}^{\alpha} \text { t.s. } H^{\alpha} \text { t.s. } C_{a}^{\alpha} \text { t.s. } C_{1}^{\alpha} \text { t.s. } \Gamma_{b}^{\alpha} \text { t.s. } C_{b}^{\alpha} \text {. }
\end{aligned}
$$

4. The exponential method. Basu in [3] denotes by $J^{\alpha}$ the Hausdorff matrix corresponding to the generating sequence $\exp \left(-\alpha n^{1 / 2}\right), \alpha>0$. He shows that the total strength of $J^{\alpha}$ increases with $\alpha$ and compares $J^{\alpha}$ totally with the Hölder method $H^{\alpha}$. It is the purpose of this section to consider similar problems for the more general method $J_{a}^{\alpha}$, where $J_{a}^{\alpha}$ is the Hausdorff method with generating sequence $\exp \left(-\alpha n^{a}\right), \alpha>0,0<a<1$.

From $\left[4\right.$, pp. 269-270] we have the results that $J_{a}^{\alpha}$ is totally regular and the proof of the following.

THEOREM 6. Let $0<a<1$. Then, for $0<\alpha<\beta$, J $J_{a}^{\alpha}$ t.s. $J_{a}^{\beta}$.

THEOREM 7. For $\alpha>0,0<a<d<1$, $J_{a}^{\alpha}$ n.t.s. $J_{d}^{\alpha}$ and $J_{d}^{\alpha}$ n.t.s. $J_{a}^{\alpha}$.

Proof. Let $\mu(t)=\exp \left[\alpha\left(t^{d}-t^{a}\right)\right]$. Since $0<a<d<1, t^{d}-t^{a}<0$ for $0<t<1$ and $t^{d}-t^{a}>0$ for $t>1$. Therefore, neither $\mu(t)$ nor its reciprocal is totally monotone.

THEOREM 8. Let $0<a<1, \alpha, \beta, c>0$. Then, for $\beta \geqq \alpha \Gamma(1-a) a^{a-1}(c e)^{-a}$, $J_{a}^{\beta}$ t.s. $\Gamma_{c}^{\alpha}$.

Proof. Let

$$
\mu(t)=\frac{(t+c)^{\alpha} e^{-\beta t^{a}}}{c^{\alpha}}
$$

and $\sigma(t)=\log \mu(t)$. Then

$$
(-1) \sigma^{\prime}(t)=\frac{a \beta}{t^{1-a}}-\frac{\alpha}{t+c} .
$$

If one shows that $(-1) \sigma^{\prime}(t)$ is a totally monotone function for $t \geqq 1$, then $\mu(t)$ will be totally monotone for $t \geqq 1$; i.e., $\Delta^{n} \mu(k) \geqq 0$ for $k=1,2, \cdots$; $\mathrm{n}=0,1,2, \cdots$. By $[8$, Theorem $8, \mathrm{p} .523]$ it then follows that $\mu(n)$ is a totally monotone sequence.

For

$$
(-1) \sigma^{\prime}(t+1)=\frac{a \beta}{(t+1)^{1-a}}-\frac{\alpha}{t+1+c}, \quad(t \geqq 0)
$$

the corresponding mass function is 


$$
\phi(u)=a \beta \chi(u)-\frac{\alpha u^{1+c}}{1+c}
$$

where

$$
\chi(u)=\frac{1}{\Gamma(1-a)} \int_{0}^{u}\left(\log \frac{1}{t}\right)^{-a} d t .
$$

For $0<u<1$,

$$
\phi^{\prime}(u)=\frac{a \beta\left(\log \frac{1}{u}\right)^{-a}}{\Gamma(1-a)}-\alpha u^{c},
$$

and $\phi^{\prime}(u)$ will be nonnegative if

$$
\frac{\left(\log \frac{1}{u}\right)^{-a}}{u^{c}} \geqq \frac{\alpha \Gamma(1-a)}{a \beta} .
$$

The minimum value of the left-hand side of the above inequality, for $0<u<1$, occurs at $u=e^{-(a / c)}$. Hence $\phi^{\prime}(u)$ will be nonnegative, provided

$$
\frac{(a / c)^{-a}}{e^{-a}} \geqq \frac{\alpha \Gamma(1-a)}{a \beta} ;
$$

i.e.,

$$
\beta \geqq \frac{\alpha \Gamma(1-a) a^{a-1}}{(c e)^{a}} .
$$

Theorem 4 (ii) of $[3$, p. 305$]$ is a special casel of the above theorem with $c=1, a=1 / 2$.

Comparing Theorem 8 above with list (R) of $\S 3$ for $\alpha>1$, we see that the $J_{a}^{\beta}$ method, with the appropriate restriction in $\beta$, is totally stronger than all of the the other methods listed. However, for $0<\alpha<1$, it is immediately apparent that we need to discuss the total relative strengths of $J_{a}^{\beta}$ and $C_{c}^{\alpha}$ for $0<c<1$. Let

$$
\mu_{n}=\frac{\Gamma(c+n+\alpha) \Gamma(c)}{\Gamma(c+\alpha) \Gamma(n+c)} e^{-\beta n^{a}} .
$$

Using the method of Theorem 1 of this paper, let

Then

$$
f(t)=\frac{\Gamma(c+t+\alpha) \Gamma(c)}{\Gamma(c+\alpha) \Gamma(t+c)} e^{-\beta t^{a}} .
$$

$$
g(t)=\frac{f(t)}{f(t+1)}=\frac{(t+c) e^{\beta\left[(t+1)^{a}-t^{a}\right]}}{(t+c+\alpha)}
$$

and

$$
h(t)=(-1) \frac{g^{\prime}(t)}{g(t)}=a \beta\left[\frac{1}{t^{1-a}}-\frac{1}{(t+1)^{1-a}}\right]-\left[\frac{1}{t+c}-\frac{1}{t+c+\alpha}\right] .
$$


The mass function corresponding to $h(t+1)$ for all $t \geqq 1$ is

where

$$
\phi(u)=a \beta\left[\chi_{1}(u)-\chi_{2}(u)\right]-\left[\frac{u^{c+1}}{c+1}-\frac{u^{c+\alpha+1}}{c+\alpha+1}\right], \quad 0 \leqq u \leqq 1,
$$

$$
\chi_{j}(u)=\frac{1}{j^{1-a} \Gamma(1-a)} \int_{0}^{u} t^{j-1}\left\{\log \frac{1}{t}\right\}^{-a} d t, \quad j=1,2 .
$$

For $0<u<1$,

$$
\begin{aligned}
\phi^{\prime}(u) & =a \beta\left[\frac{\left(\log \frac{1}{u}\right)^{-a}}{\Gamma(1-a)}-\frac{u\left(\log \frac{1}{u}\right)^{-a}}{\Gamma(1-a) 2^{1-a}}\right]-u^{c}+u^{c+\alpha} \\
& =\frac{a \beta\left(\log \frac{1}{u}\right)^{-a}}{2^{1-a} \Gamma(1-a)}\left(2^{1-a}-u\right)-u^{c}\left(1-u^{\alpha}\right) .
\end{aligned}
$$

Since $0<a<1,0<c<1,0<u<1,2^{1-a}-u \geqq 1-u^{\alpha}$. Therefore $\phi^{\prime}(u)$ will be nonnegative, provided

$$
\frac{a \beta\left(\log \frac{1}{u}\right)^{-a}}{2^{1-a} \Gamma(1-a)} \geqq u^{c}
$$

i.e.,

$$
\frac{\left(\log \frac{1}{u}\right)^{-a}}{u^{c}} \geqq \frac{2^{1-a} \Gamma(1-a)}{a \beta} .
$$

But the left hand side of the above inequality occured in the proof of Theorem 8, and its minimum occurs at $u=e^{-(c / a)}$. Therefore the condition

$$
\beta \geqq 2^{1-a} \Gamma(1-a) a^{a-1}(c e)^{-a}
$$

is sufficient to guarantee that $\phi(u)$ is a totally monotone mass function and hence that $\mu(t)$ is totally monotone for $t \geqq 1$. As in the proof of Theorem 8 it then follows that $\mu(t)$ is totally monotone. We have therefore proved the following theorem.

THEOREM 9. Let $0<a<1$, $0<c<1$, $0<\alpha<1$, $\beta>0$. Then, for $\beta \geqq 2^{1-a} \Gamma(1-a) a^{a-1}(c e)^{-a}, J_{a}^{\beta}$ t.s. $C_{c}^{\alpha}$.

The proof of Theorem 8 , in addition to generalizing [3, Theorem 4] is also substantially simpler. However, it cannot be used to prove Theorem 9 because of the presence of the logarithmic derivative of the gamma function. The proof of Theorem 9 can be used to prove a weaker form of Theorem 8. Thus, Theorem 9 may not be the best possible result for the comparison it deals with. 
Theorems 8 and 9 have a restrictive condition involving the parameters. Therefore it would appear that, for some values of the parameters, total comparison is not possible. Since the sequences corresponding to the methods $J_{a}^{\beta} / \Gamma_{c}^{\alpha}$ and $J_{a}^{\beta} / C_{c}^{\alpha}$ both tend to zero, it is clear that a comparison such as $\Gamma_{c}^{\alpha}$ t.s. $J_{a}^{\beta}$ or $C_{c}^{\alpha}$ t.s. $J_{a}^{\beta}$ is impossible. It remains, therefore, to determine if there are values of the parameters for which $J_{a}^{\beta}$ n.t.s. $\Gamma_{c}^{\alpha}$ and $J_{a}^{\beta}$ n.t.s. $C_{c}^{\alpha}$. The latter relationship is quite difficult to deal with, so we conclude with the following theorem.

TheOREM 10. Let $\alpha, \beta, c>0,0<a<1$. Then, for

$$
\beta<\alpha a^{a-1}(2-a)^{2-a}\left[4 c^{a}(1-a)\right]^{-1}, J_{a}^{\beta} \text { n.t.s. } \Gamma_{c}^{\alpha} \text {. }
$$

The theorem is proved in the same way as [3, Theorem 5] and therefore the proof will be omitted here.

I wish to express my thanks to M. S. Ramanujan who sent me his unpublished results dealing with the total relative strengths of some of the methods of this paper. The results communicated to me were the statements of the following theorems: 2 for $\alpha$ positive, 3 (iii), 4 , and 8 and 10 with $\alpha=1 / 2$.

\section{REFERENCES}

1. S. K. Basu, On the total relative strength of the Hölder and Cesàro methods, Proc. London Math. Soc. 50 (1948-1949), 447-462.

2. - On hypergeometric summability involving infinite limits, Proc. Amer. Math. Soc 5 (1954), 226-238.

3. - On comparison of the total strength of some Hausdorff methods, Math. Z. 67 (1957), 303-309.

4. G. Hardy, Divergent series, Oxford, 1949.

5. F. Hausdorff, Summationsmethoden und Momentfolgen, Math. Z. 9 (1921), 74-109. (p. 95).

6. —_ Über das Momentproblem für ein endliches interval, Math. Z. 16 (1923), 220-248.

7. B. E. Rhoades, Total comparison among some totally regular Hausdorff methods, Math. Z. 72 (1960), 463-466.

8. - Some properties of totally coregular matrices, Illinois J. Math. 4 (1960), 518-525.

9. - Hausdorff summability methods, Trans. Amer. Math. Soc. 101 (1961), 396-425.

10. H. S. Wall, Continued fractions and totally monotone sequences, Trans. Amer. Math. Soc. 48 (1940), 165-184.

11. D. V. Widder, The Laplace transform, Princeton Univ. Press, Princeton, N. J., 1946.

LAFAyetTe College,

Easton, Pennsylvania 